\title{
The impact of early growth patterns and infant feeding on body composition at 3 years of age
}

\author{
Katrine T. Ejlerskov ${ }^{1 *}$, Line B. Christensen ${ }^{2}$, Christian Ritz ${ }^{2}$, Signe M. Jensen ${ }^{2}$, Christian Mølgaard ${ }^{2}$ \\ and Kim F. Michaelsen ${ }^{2}$ \\ ${ }^{1}$ The National Food Institute, Technical University of Denmark, Mørkhøj Bygade 19, 2860 Søborg, Denmark \\ ${ }^{2}$ Department of Nutrition, Exercise and Sports, Faculty of Science, University of Copenhagen, 1958 Frederiksberg, Denmark
}

(Submitted 19 November 2014 - Final revision received 5 March 2015 - Accepted 2 April 2015 - First published online 1 July 2015)

\begin{abstract}
Early excessive weight gain is positively associated with later obesity, and yet the effect of weight gain during specific periods and the impact of infant feeding practices are debated. The objective of the present study was to examine the impact of weight gain in periods of early childhood on body composition at 3 years, and whether infant feeding modified the relationship between early growth and body composition at 3 years. We studied 233 children from the prospective cohort study, SKOT (in Danish: Småbørns Kost og Trivsel). Birth weight $z$-scores (BWZ) and change in weight-for-age $z$-scores (WAZ) from 0 to 5,5 to 9,9 to 18 and 18 to 36 months were analysed for relations with body composition (anthropometry and bioelectrical impedance) at 3 years by multivariate regression analysis. BWZ and change in WAZ from 0 to 5 months were positively associated with BMI, fat mass index (FMI) and fat-free mass index (FFMI) at 3 years. Full breastfeeding for 6 months (compared to less than 1 month) eliminated the effect of early growth $(P=0 \cdot 01)$. Full breastfeeding for 6 months (compared to less than 1 month) also eliminated the positive relation between BWZ and FMI $(P=0 \cdot 009)$. No effect modification of infant feeding was found for FFMI. In conclusion, high birth weight and rapid growth from 0 to 5 months were associated with increased FMI and FFMI at 3 years. Longer duration of full breastfeeding reduced the effect of birth weight and early weight gain on fat mass.
\end{abstract}

Key words: Early growth patterns: Infant feeding: Body composition: Fat mass index: Childhood

Many studies have shown highly significant associations between weight gain during the first months or years of life and later adiposity ${ }^{(1,2)}$. The first 4-9 months of life have been identified as a sensitive period for the influence of rapid growth on fat mass (FM) at later ages ${ }^{(3-5)}$ and later risk of obesity ${ }^{(6-8)}$. Alternatively, other studies have found excessive weight gain in the period $2-6$ years ${ }^{(5,9)}$ and no specific period (from birth to 15 years) at all ${ }^{(10)}$ to be of particular importance.

Nutrition in infancy is thought to be particularly influential on later risk of obesity, as growth patterns and changes in body composition in early infancy are closely linked with feeding patterns ${ }^{(3,11-13)}$. Breastfed infants show slower weight gains in the first year of life ${ }^{(11,12)}$, and have lower FM at 12 months compared to formula-fed infants ${ }^{(13)}$. Three meta-analyses have shown breastfeeding to be associated with a small but consistently reduced risk of obesity determined by $\mathrm{BMI}^{(14-16)}$, while breastfeeding does not seem to reduce mean $\mathrm{BMI}^{(17)}$. However, less consistency has been found with regard to the relationship between infant feeding and FM and fat-free mass (FFM) at later ages. Three studies have found an inverse relationship between duration of breastfeeding and FM at 4 years $^{(18)}$, 9-10 years ${ }^{(19)}$ and 16 years of age ${ }^{(20)}$; one study found an inverse effect in 9-year-old girls but not boys ${ }^{(21)}$; three studies found no differences at 2 years $^{(22)}, 5$ years ${ }^{(23)}$ and 18 years of age ${ }^{(24)}$.

Supporting the theory that early overnutrition leads to increased risk of obesity at later ages, two randomised controlled trials demonstrated increased growth rates and higher FM at 5-8 years of age in a group of children born small for gestational age who had received nutrient-enriched formula $v$. control formula until 6-9 months of age ${ }^{(3)}$. However, fewer studies have focused on the relation between the timing of the introduction of solids and later risk of obesity, and the findings that exist are inconsistent ${ }^{(25,26)}$. Early introduction to solids has been found to be associated with higher energy intake ${ }^{(27,28)}$, and earlier introduction to unhealthy foods ${ }^{(29)}$.

Abbreviations: BAZ, BMI-for-age $z$-score; BIA, bioelectrical impedance analysis; BWZ, birth weight $z$-score; DXA, dual-energy X-ray analysis; FFM, fat-free mass; FFMI, fat-free mass index; FM, fat mass; FMI, fat mass index; HAZ, height/length-for-age $z$-score; SKOT, Småbørns Kost og Trivsel; WAZ, weight-for-age $z$-score; WFH, weight-for-height/length $z$-score.

*Corresponding author: K. T. Ejlerskov, email ktan@food.dtu.dk 
Breastfeeding, formula feeding and complementary feeding practices are three highly related components, and it can be difficult to distinguish which component is responsible for certain effects. Therefore, both breastfeeding and information on complementary feeding should be included in analyses of effects of early diet and body composition in later childhood $^{(18,23)}$. Although large variations in body composition exist within a given $\mathrm{BMI}^{(30)}$, most studies of both early growth and early feeding have used BMI or BMI standard deviation scores as indirect measures of adiposity at later age stages ${ }^{(1,2,14-17)}$. In the present study, however, we supplement anthropometric measurements and BMI with fat mass index (FMI) and fat-free mass index (FFMI) estimated from bioelectrical impedance analysis (BIA). In order to elucidate the mechanisms behind rapid growth and to improve prevention strategies of childhood obesity, it is important to identify the age window that is most impacted in this regard, and modifiable factors that influence growth in this period. The main aim of the present study was to undertake a detailed investigation of how weight gain in four periods, spanning birth to 3 years of age, influences body composition at 3 years of age. Our secondary aim was to examine the impact of breastfeeding and age of introduction to solids on FMI and FFMI.

\section{Subjects and methods}

\section{Study design and participants}

The data of the present study is drawn from the SKOT cohort (in Danish: Småbørns Kost og Trivsel), a prospective observational cohort study that monitored healthy Danish children at 9, 18 and 36 months of age. The present study was previously described in detail by Madsen et al. ${ }^{(31)}$. In brief, the criteria for the study included singleton term infants (at a gestational age of 37-42 weeks) without any diseases expected to affect growth or food intake. Recruitment took place from April 2007 to May 2008, with 2211 families randomly selected from the National Danish Civil Registry to be invited to participate in the present study. Out of these, 330 families accepted the invitation, and were enrolled in the study. The 36-month examinations took place from October 2009 to October 2010.

\section{Anthropometry}

Birth weight and weight at 5 months (measured by midwives and general medical practitioners, respectively) were obtained from health records kept by the parents of the study participants. All other anthropometric measurements, including weight, height and skin folds, were obtained from physical examinations of 9-month ( \pm 2 weeks), 18-month ( \pm 1 month) and 36-month ( \pm 3 months) children at the Department of Nutrition, Exercise, and Sports, Copenhagen, Denmark. The procedures for the anthropometric measurement of 9- and 18-month children were described previously by Madsen et al. ${ }^{(32)}$. At 36 months, naked body weight was measured to the nearest $0.1 \mathrm{~kg}$ on an annually calibrated digital scale (Tanita WB-100MA; Tanita Corporation), and height was measured by a stationary digital stadiometer (235 Heightronic Digital Stadiometer; QuickMedical) to the nearest $0.01 \mathrm{~cm}$. Triceps and subscapular skin folds were measured by a Harpenden skinfold calliper (Chasmors Limited) and recorded to the nearest $0 \cdot 1 \mathrm{~mm}$. Except for weight, all measurements were performed in triplicate, and averages were used in analysis. Following standardised procedures, four well-trained observers conducted the examinations. Weight and height/length were entered into the software WHO Anthro $2005^{(33)}$ to achieve sex-specific $z$-scores. The number of overweight and obese children at 3 years of age was determined according to the cut-off values of the International Obesity Task Force ${ }^{(34)}$ and according to WHO growth standards ${ }^{(35)}$.

\section{Body composition assessment at 3 years of age}

Body composition at 36 months was measured using BIA, described in detail by Ejlerskov et al. ${ }^{(36)}$. Whole body resistance, reactance and impedance were measured with the child in a supine position using a single-frequency $(50 \mathrm{kHz})$ tetrapolar BIA (Quantum III; RJL Systems). On the right foot, the signal electrode (LMP3 Diagnostic Tab Electrodes; Kendall, Covidien) was placed over the distal portion of the second metatarsal (the base of the second toe), and the detecting electrode was placed at the anterior ankle on an imaginary line bisecting the medial malleolus. On the right hand, the signal electrode was placed above the metacarpophalangeal joint of the middle finger. The detecting electrode on the right hand was placed on an imaginary line bisecting the ulnar head, as specified by the manufacturer. These measurements were performed twice consecutively, and the mean values of resistance, reactance and impedance were registered. A prediction equation for the FFM of the study's cohort of 3-year-old children was developed in advance using BIA, height and weight, with dual-energy X-ray analysis (DXA) as a reference method ${ }^{(36)}$. In short, high quality DXA scans were achieved for a subgroup of the SKOT children ( $n$ 101), and a prediction equation was obtained via linear regression models using a 10-fold cross validation approach with BIA, weight, height and sex as independent variables (adjusted $\left.R^{2} 0 \cdot 84\right)^{(36)}$. FFM and FM were calculated using the following equations:

$$
\begin{aligned}
\text { FFM }= & 327.2 \text { RI }+223.8 \text { weight }+76.8 \text { height }+417.6 \\
& \text { sex }-2784.4 \\
& \text { FM }=(\text { weight } \times 0.981+0.374) \times 1000-\text { FFM }
\end{aligned}
$$

where FFM and FM are in grams, RI is the resistance index (height $(\mathrm{cm})^{2} /$ resistance $(\Omega)$, weight is digital weight computed in $\mathrm{kg}$, height is in $\mathrm{cm}$, and sex is recorded as male $=1$ and female $=0$.

\section{Feeding patterns and other information}

At the 9-month examination, the participants' parents filled out questionnaires on annual household income, age, height and weight of parents, infant gestational age at birth, gestational 
weight gain and smoking during pregnancy. In all three examinations, parents filled out follow-up questionnaires with updated information regarding their educational status, from which a variable of the mothers' educational levels at the time of the 3-year examination was generated. At the 36-month examination, we measured the height and weight of the accompanying parent using the same measuring equipment as used for the children. Data on feeding patterns, including duration of full and partial breastfeeding and age of introduction to complementary foods were obtained in interviews at each examination. Full breastfeeding was defined as receiving only breast milk, water and vitamins, though allowed for exceptional bottle feeding (e.g. if a child had been babysat for a single night), which increased the duration of full breastfeeding for fifteen infants. Partial breastfeeding was defined as a baby receiving some breast milk along with other food, weaning foods or formula milk. Age of introduction to solids was defined as the earliest age in months at which an infant first received one of nineteen food categories. Information on formula intake $(\mathrm{g} / \mathrm{d})$ at 9 months was derived from pre-coded dietary records developed for infants as described in Gondolf et al. ${ }^{(37)}$.

\section{Ethics}

The parents of all participants received verbal and written information about the study and written consent was obtained from all. The study was approved by The Committees on Biomedical Research Ethics for the Capital Region of Denmark (H-KF-2007-0003).

\section{Statistical analysis}

The study sample only included data with complete records on FM and FFM. Missing values were considered missing at random, and available-cases analyses were carried out.

To account for natural variation in FM and FFM due to body size, we calculated FMI as FM/height $\left(\mathrm{kg} / \mathrm{m}^{2}\right)$, and FFMI as FFM/height $\left(\mathrm{kg} / \mathrm{m}^{2}\right)^{(38)}$. Linear regression confirmed that FMI and FFMI were not associated with height $(P>0.5)$. In the absence of well-established reference data for FM or FMI to identify cut-off values for overweight-ness in young children, we grouped FMI and FFMI according to sex-specific quartiles.

Differences of weight-for-age $z$-scores (WAZ), height/lengthfor-age $z$-scores (HAZ), BMI-for-age $z$-scores (BAZ) and weight-for-height/length $z$-scores (WFH) in the study sample were evaluated to the WHO standard using one-sample $t$ tests. Differences in the feeding patterns between sexes were assessed either using two-sample $t$ tests or, if not normally distributed, Wilcoxon rank tests. Anthropometry at 3 years of age, parental characteristics and infant feeding practices were evaluated according to FMI and FFMI quartiles using mean values and standard deviations, and medians and interquartile ranges (if not normally distributed). Testing of trends across quartiles of FMI and FFMI was based on linear regression.

Associations between body composition at 3 years of age and weight gain in four age intervals ( $0-5$ months, 5-9 months, 9-18 months and 18-36 months) were analysed using multiple linear regression. Body composition outcomes were regressed on change in WAZ in each of the four age intervals in both a simple model, only adjusting for sex and birth weight $z$-score (BWZ), and in a fully adjusted model controlling for sex, BWZ, household income, educational level of mother, smoking during pregnancy, gestational weight gain and parental BMI or height or weight according to the outcome of interest. The educational level of the mother was grouped into five categories: 'no education above school level', 'trainee or vocational education', 'short academic education $<3$ years of age', 'academic education 3-4 years' and 'long academic education $>4$ years'. Data on household incomes were collected in categories ranging from 'less than 200000 Danish Kroner' to 'more than 800000 Danish Kroner', with fourteen intervals of 50000 Danish Kroner (approximately 8700 US\$) in between, and these groupings were used as quantitative variables in the analyses. As only few mothers reported smoking in pregnancy, this variable was included as a dichotomous one (yes/no). Model assumptions were evaluated using residual and normal probability plots. Robust standard errors were employed, in case substantial departures were found.

For each quartile of FMI, the development of mean WAZ across the four time points was visualised in scatter plots. The same was done for each quartile of FFMI. Differences between quartiles of mean WAZ over time were evaluated using linear mixed models while controlling for sex, household income, educational level of mother, smoking during pregnancy, gestational weight gain and parental BMI, including child-specific random effects.

To identify the impact of birth weight and early growth on FMI and FFMI, we grouped birth weight into four categories: '<3000 g' ( $n$ 23), '3000-3499 g' ( $n$ 92), '3500-3999 g' ( $n$ 83) and ' $\geq 4000$ g' ( $n$ 35). Only two children were born with a weight below $2500 \mathrm{~g}$. Change in WAZ from 0 to 5 months was also divided into four categories: ' $<-0.67$ ', ' $-0.67-0$ ', '0-0.67' and ' $>0.67$ '. A change in WAZ exceeding 0.67 represents upward centile crossing at standard growth curves, which is a threshold that has been found clinically relevant ${ }^{(39)}$. Differences in the proportion of children in the highest quartile of FMI and FFMI across the categories were tested using $\chi^{2}$ test or Fisher's exact test.

Associations between FMI or FFMI, duration of full and partial breastfeeding, and age of introduction to solids were assessed, using multiple regression in simple models adjusted only for sex, and in fully adjusted models while controlling for BWZ, WAZ change from 0 to 5 months, educational level of mother, gestational weight gain and maternal BMI. We included the combined effects corresponding to the BWZmodified effect of full breastfeeding, the BWZ-modified effect of age of introduction to solids, the effect modification of full breastfeeding by WAZ change from 0 to 5 months, and the effect modification of age of introduction to solids by WAZ change from 0 to 5 months in a fully adjusted regression model with FMI or FFMI as outcome variables. For these analyses, full breastfeeding was categorised as '< 1 month' (<31 d, n 36), '1-3 months' (31-120 d, $n$ 39), '4-5 months' (121-180d, $n$ 137) and ' 6 months' (>180 d 
(range 183-197d), $n$ 21). Age of introduction to solids was grouped as ' $3-4$ months' ( $n$ 138), ' 5 months' ( $n$ 63) and '6 months' ( $n$ 32). Backwards stepwise elimination was used for removing non-significant combined effects one at a time by means of likelihood ratio tests. The same procedure was applied to a similar model with the variables of partial breastfeeding used in interaction terms ( $<4$ months' $(<121 \mathrm{~d}$, $n$ 30), '4-5 months' (121-182d, $n$ 30), '6-8 months' (183-273d, $n$ 60), '9-11 months' (274-364d, $n$ 56) and ' $\geq 12$ months' (>365 d, $n$ 54)).

Analyses were carried out using STATA version 11.0 (StataCorp LP). The significance level was set at $\alpha=0.05$.

\section{Results}

Out of the 330 children initially recruited for SKOT, 263 (79.7\%) completed the 36-month examination. FFM and FM were calculated for the 233 children with complete BIA, height and weight data. Children without BIA data were taller at 3 years of age than children with complete BIA data ( $P=0.008$, data not shown), and yet no differences were seen in terms of weight and BMI at 3 years of age. Data for weight and length at 5 months was missing for sixty-six children. A comparison of the children with or without 5-month values showed no differences in weight and length at birth and 9 months of age (data not shown). Selected characteristics of parents are presented in Table 1.

\section{Sample characteristics}

The mean WAZ of the SKOT children was above average compared with WHO growth standards in all examinations (all $P<0.001$; Table 2). HAZ was measured at birth and 5 months by midwives and practitioners and was substantially higher than the WHO standards, most likely due to inaccurate length measures in the primary healthcare sector. At 3 years of age, nineteen children were overweight ( $8.2 \%)$, and none was obese according to the International Obesity Task Force criteria. Forty-six children (19.7\%) were at risk of becoming overweight (with a BAZ of above $1 \mathrm{SD}$ ), four children $(1.7 \%)$ were overweight with a BAZ above $2 \mathrm{SD}$ and none obese according to the WHO growth standards. A total of 158 children $(67.8 \%)$ were fully breastfed for 4 months or more, while ninety-nine children (42.5\%) were still partially breastfed at 9 months. Five children (2\%) were introduced to solids earlier than 4 months, $133(57 \%)$ at 4 months, 63 children $(27 \%)$ at 5 months and 32 children (14\%) at 6 months.

Feeding patterns were found not to differ between boys and girls (all $P>0.3$, data not shown); however, infants who were no longer fully breastfed at 4 months were introduced to solids earlier than infants fully breastfed at 4 months $(P<0.001)$ (data not shown). No trends across FMI or FFMI quartiles were found for the majority of the infant feeding variables (Tables 3 and 4), and yet a negative trend across FMI quartiles was seen for the number of children fully breastfed at 4 months $(P=0.028)$ (Table 3$)$. There was also a trend of higher maternal BMI in the higher FMI quartiles $(P=0.07)$. Maternal age, height, gestational weight gain, smoking
Table 1. Baseline characteristics

(Median values and 25th-75th percentiles, number of participants and percentages)

\begin{tabular}{|c|c|c|c|}
\hline Characteristics & $n$ & Median & $\begin{array}{l}\text { 25th-75th } \\
\text { percentile }\end{array}$ \\
\hline \multicolumn{4}{|l|}{ Mother } \\
\hline Weight $(\mathrm{kg})$ & 233 & $65 \cdot 3$ & $59-72$ \\
\hline Height (m) & 233 & 1.68 & $1.64-1.72$ \\
\hline $\mathrm{BMI}$ & 233 & 22.9 & $21 \cdot 0-25 \cdot 1$ \\
\hline Gestational weight gain & 226 & 14.5 & $12 \cdot 0-17 \cdot 0$ \\
\hline \multicolumn{4}{|l|}{ Father } \\
\hline Weight (kg) & 226 & $83 \cdot 8$ & $75 \cdot 9-92 \cdot 0$ \\
\hline Height (m) & 228 & 1.82 & $1.78-1.86$ \\
\hline \multirow[t]{2}{*}{$\mathrm{BMI}$} & 226 & $25 \cdot 0$ & $23 \cdot 4-27 \cdot 4$ \\
\hline & $n$ & \multicolumn{2}{|r|}{$\%$} \\
\hline \multicolumn{4}{|l|}{ Smoking during pregnancy } \\
\hline Smokers & 13 & \multicolumn{2}{|r|}{$5 \cdot 6$} \\
\hline Non-smokers & 220 & \multicolumn{2}{|r|}{94.4} \\
\hline \multicolumn{4}{|c|}{ Educational level of the mother } \\
\hline $\begin{array}{l}\text { No education } \\
\text { above school level }\end{array}$ & 10 & \multicolumn{2}{|r|}{$4 \cdot 3$} \\
\hline $\begin{array}{l}\text { Trainee or vocational } \\
\text { education }\end{array}$ & 18 & \multicolumn{2}{|r|}{$7 \cdot 7$} \\
\hline $\begin{array}{l}\text { Short academic } \\
\text { education }<3 \text { years }\end{array}$ & 28 & \multicolumn{2}{|r|}{$12 \cdot 0$} \\
\hline $\begin{array}{l}\text { Academic education } \\
3-4 \text { years }\end{array}$ & 82 & \multicolumn{2}{|r|}{$35 \cdot 2$} \\
\hline $\begin{array}{l}\text { Long academic } \\
\text { education }>4 \text { years }\end{array}$ & 95 & \multicolumn{2}{|r|}{$40 \cdot 8$} \\
\hline \multicolumn{4}{|c|}{ Family income (Danish Kroner) } \\
\hline$<200 \cdot 000$ & 10 & & $4 \cdot 3$ \\
\hline $200 \cdot 000-399.999$ & 35 & & $15 \cdot 0$ \\
\hline $400 \cdot 000-599.999$ & 35 & & $15 \cdot 0$ \\
\hline $600 \cdot 000-799.999$ & 71 & & $30 \cdot 5$ \\
\hline$>800 \cdot 000$ & 77 & & $33 \cdot 0$ \\
\hline Unknown & 5 & & $2 \cdot 1$ \\
\hline
\end{tabular}

during pregnancy, parental educational level, paternal height and BMI, and household incomes were not related to the FMI quartiles (all, $P>0 \cdot 13$, data not shown). Positive trends across FFMI quartiles were seen for parental BMI (both $P<0.02$ ), while negative trends were seen for parental education (both $P<0.05$, data not shown). Maternal age, height, gestational weight gain, smoking during pregnancy, paternal height and household incomes were not related to the FFMI quartile (all, $P>0 \cdot 18$, data not shown).

\section{Association between early weight gain and body composition at 3 years of age}

BWZ and change in WAZ from 0 to 5 months were found to be positively related to height, weight, BMI, FMI and FFMI at 3 years of age in both the simple and adjusted models (Table 5). In addition to this, change in WAZ from 0 to 5 months was positively associated with the sum of skin folds $(P<0 \cdot 001)$. The different growth patterns over time for the mean WAZ according to the FMI quartiles are shown in Fig. 1(a). There was significant interaction between the FMI quartiles and time $(P<0 \cdot 001)$, with the most evident difference existing between growth patterns from 0 to 5 months. Controlling for the educational level of the mother, smoking 
Table 2. Anthropometric characteristics in the Småbørns Kost og Trivsel (SKOT) cohort according to WHO $z$-scores ${ }^{(35) *}$

(Mean values and ranges (minimum-maximum))

\begin{tabular}{lrrr}
\hline & Mean & Range & $P$ \\
\hline At birth (n 233)† & & & \\
WAZ & 0.48 & $-1.98-2.71$ & $<0.001$ \\
HAZ & 1.39 & $-3.84-4.29$ & $<0.001$ \\
WFH & -0.95 & $-4.71-2.54$ & $<0.001$ \\
BAZ & -0.35 & $-3.99-2.63$ & $<0.001$ \\
At 5 months $(n$ 167) & & & \\
WAZ & 0.32 & $-2.00-3.29$ & $<0.001$ \\
HAZ & 1.21 & $-1.12-4.21$ & $<0.001$ \\
WFH & -0.40 & $-2.57-2.89$ & $<0.001$ \\
BAZ & -0.48 & $-2.62-2.88$ & $<0.001$ \\
At 9 months $(n$ 233) & & & \\
WAZ & 0.40 & $-1.62-3.00$ & $<0.001$ \\
HAZ & 0.25 & $-1.94-2.84$ & $<0.001$ \\
WFH & 0.43 & $-1.52-4.21$ & $<0.001$ \\
BAZ & 0.35 & $-1.75-4.37$ & $<0.001$ \\
At 18 months $(n$ 229) & & & \\
WAZ & 0.41 & $-2.02-2.58$ & $<0.001$ \\
HAZ§ & 0.11 & $-2.91-2.53$ & 0.07 \\
WFH§ & 0.49 & $-1.86-3.00$ & $<0.001$ \\
BAZ§ & 0.49 & $-1.69-3.14$ & $<0.001$ \\
At 36 months $(n$ 233) & & & \\
WAZ & 0.17 & $-2.06-2.04$ & $<0.001$ \\
HAZ & -0.05 & $-2.09-2.36$ & 0.34 \\
WFH & 0.28 & $-1.76-2.36$ & $<0.001$ \\
BAZ & 0.27 & $-1.78-2.44$ & $<0.001$ \\
\hline
\end{tabular}

WAZ, weight-for-age $z$-score; HAZ, height/length-for-age $z$-score; WFH, weight-forheight/length $z$-score; BAZ, BMI-for-age $z$-score.

*Differences between WAZ, HAZ, WFH and BAZ in the study sample, as well as the WHO standards, were evaluated by means of one-sample $t$ tests.

$\uparrow$ Measured by midwives at the hospital.

$\ddagger$ Measured by general medical practitioners.

$\S$ Missing values for three children.

during pregnancy, gestational weight gain and parental BMI, differences between the quartiles showed that the mean WAZ of the highest FMI quartile was significantly higher than that of the other FMI quartiles at 5 months $(P<0.002)$ and 9 months $(P<0.05)$. At 18 months, only the WAZ of the first and second FMI quartiles were significantly lower than the highest $(P<0.002)$. At 36 months, the third FMI quartile was only just lower than the fourth FMI quartile $(P=0.08)$, and yet the others remained even lower $(P<0 \cdot 001)$. Growth patterns over time did not differ between the FFMI quartiles $(P=0 \cdot 17)$, but the mean WAZ of the fourth quartile was significantly higher than all the other quartiles $(P<0 \cdot 001)$ (Fig. 1(b)).

\section{Fat mass index and fat-free mass index according to birth weight and early weight gain}

The probability of being in the highest quartile of FMI and FFMI because of birth weight and early weight gain is shown in Table 6. We categorised birth weight into four categories and analysed the share of children from each category that were placed in the highest quartile of FMI and FFMI at 3 years of age. The same approach was taken for change in WAZ from 0 to 5 months. More children with a birth weight above $4000 \mathrm{~g}$ were placed in the highest quartile of FMI compared to children with birth weights from 3000 to $3500 \mathrm{~g}$ ( $40 \%$ compared to $17 \%, P<0 \cdot 007$ ). For the group of children undergoing rapid growth in their first five months of life (change in WAZ >0.67), 49\% were placed in the highest quartile of FMI at 3 years of age, which was more than that of children with weight gains not exceeding 0.67 WAZ from 0 to 5 months (all groups, $P<0 \cdot 031$ ). A significantly larger proportion of the children with birth weights above $4000 \mathrm{~g}$, when compared to 3000-3500 g and less than $3000 \mathrm{~g}$ were found to be in the highest FFMI quartile at 3 years of age (40\% compared to 17 and $13 \%$, respectively, both $P<0 \cdot 04$ ). WAZ change from 0 to 5 months did not affect the probability of being in the highest FFMI quartile at 3 years of age.

\section{Associations between early feeding practices and body composition at 3 years of age}

Both in the unadjusted and adjusted regression analyses, full breastfeeding, partial breastfeeding, age of introduction to solids and age of introduction to cow's milk were found not to be related to FMI or FFMI at 3 years of age (data not shown). However, when testing for possible interactions between early feeding practices and early growth, we found two effect modifications of duration of full breastfeeding (Table 7). The effect of BWZ on FMI was eliminated by full breastfeeding for 6 months compared to less than 1 month (from $\beta=0.64$ to $\beta=0.02$, $P=0.009)$ (Table 7 and Fig. 2(a)). A duration of full breastfeeding for 1-3 months and 4-5 months borderline attenuated the positive effect of change in WAZ from 0 to 5 months on FMI by $41 \%$ compared to less than 1 month (from $\beta=0.73$ to $\beta=0.44, P=0 \cdot 13$ and $\beta=0 \cdot 43, P=0 \cdot 10$, respectively), while 6 months of full breastfeeding eliminated the effect of WAZ on FMI (from $\beta=0.73$ to $\beta=-0.03, P=0.010$ ) (Table 7 and Fig. 2(b)). The effect of age of introduction to solids on FMI did not interact with duration of full breastfeeding $(P>0 \cdot 25)$, and no interactions were found between sex and age of introduction to solids or duration of full breastfeeding $(P>0 \cdot 23)$.

Neither age of introduction to solids nor duration of partial breastfeeding modified the effect of BWZ, or change in WAZ from 0 to 5 months on FMI $(P>0 \cdot 3)$. No interactions were found between BWZ, WAZ change from 0 to 5 months and early feeding practice on FFMI $(P>0 \cdot 6)$

\section{Discussion}

In this prospective cohort, we found that high birth weight and rapid growth in the first 5 months of life independently affected body composition and measures of adiposity at 3 years of age, while there were no effects of weight gain in the following age periods. Full breastfeeding for 6 months considerably attenuated the effect of birth weight and weight gain from 0 to 5 months on FMI at 3 years compared to less than 1 month. The inherent limitations of an observational study granted, the results of the present study indicate that full breastfeeding for 6 months, which is a modifiable factor, can attenuate the effect of birth weight and early growth on FM at 3 years of age.

After controlling for parental height, weight or BMI, gestational weight gain, mother's educational level and smoking during pregnancy, the results show that birth weight and 
Table 3. Characteristics of the Småbørns Kost og Trivsel (SKOT) children according to fat mass index (FMI, $\left.\mathrm{kg} / \mathrm{m}^{2}\right)$ quartile at 3 years of age (Mean values and standard deviations, medians and interquartile ranges (IQR) (if not normally distributed), number of participants and percentages)

\begin{tabular}{|c|c|c|c|c|c|c|c|c|c|c|c|c|}
\hline & \multirow[b]{3}{*}{$n$} & & & \multicolumn{8}{|c|}{ 3-Year FMI quartile } & \multirow[b]{3}{*}{$P$ for trend } \\
\hline & & \multicolumn{2}{|c|}{ Overall } & \multicolumn{2}{|c|}{$1(n 58)$} & \multicolumn{2}{|c|}{$2(n 58)$} & \multicolumn{2}{|c|}{$3(n 59)$} & \multicolumn{2}{|c|}{$4(n 58)$} & \\
\hline & & Median & IQR & Median & IQR & Median & IQR & Median & IQR & Median & IQR & \\
\hline \multicolumn{13}{|l|}{ Anthropometric characteristics } \\
\hline$F M$ in girls $(\mathrm{kg}) \dagger$ & 122 & & & & & & & & & & & $<0.001$ \\
\hline Mean & & \multicolumn{2}{|c|}{$2 \cdot 71$} & \multicolumn{2}{|c|}{1.82} & \multicolumn{2}{|c|}{$2 \cdot 44$} & \multicolumn{2}{|c|}{3.00} & \multicolumn{2}{|c|}{$3 \cdot 61$} & \\
\hline SD & & \multicolumn{2}{|c|}{0.74} & \multirow{2}{*}{\multicolumn{2}{|c|}{0.34}} & \multicolumn{2}{|c|}{0.23} & \multicolumn{2}{|c|}{0.21} & \multirow{2}{*}{\multicolumn{2}{|c|}{0.43}} & \\
\hline FM in boys $(\mathrm{kg}) \dagger$ & 111 & & & & & & & & & & & $<0.001$ \\
\hline Mean & & \multicolumn{2}{|c|}{$2 \cdot 29$} & \multicolumn{2}{|c|}{1.56} & \multicolumn{2}{|c|}{2.03} & \multicolumn{2}{|c|}{$2 \cdot 41$} & \multicolumn{2}{|c|}{$3 \cdot 13$} & \\
\hline SD & & \multicolumn{2}{|c|}{0.65} & & & \multicolumn{2}{|c|}{$0 \cdot 16$} & & & \multicolumn{2}{|c|}{0.38} & \\
\hline FFM in girls $(\mathrm{kg}) \dagger$ & 122 & & & & & & & & & & & $<0.001$ \\
\hline Mean & & \multicolumn{2}{|c|}{11.65} & & & & & & & & & \\
\hline SD & & & & & & & & & & & & \\
\hline FFM in boys (kg) $\dagger$ & 111 & & & & & & & & & & & 0.008 \\
\hline Mean & & & 67 & & & & & & & & & \\
\hline SD & & & 1 & & & & & & & & & \\
\hline $\mathrm{BMI}\left(\mathrm{kg} / \mathrm{m}^{2}\right)$ & 233 & $15 \cdot 8$ & $15 \cdot 1-16 \cdot 6$ & $14 \cdot 8$ & $14 \cdot 3-15 \cdot 3$ & $15 \cdot 4$ & $15 \cdot 1-15 \cdot 8$ & $16 \cdot 0$ & $15 \cdot 7-16 \cdot 4$ & $17 \cdot 4$ & $16 \cdot 9-17 \cdot 9$ & $<0.001$ \\
\hline Skin folds triceps $(\mathrm{mm})$ & 224 & $9 \cdot 3$ & $8 \cdot 1-10 \cdot 4$ & $8 \cdot 2$ & $7 \cdot 6-9 \cdot 3$ & $8 \cdot 8$ & $8 \cdot 0-10 \cdot 3$ & 9.4 & $8 \cdot 3-10 \cdot 4$ & $10 \cdot 4$ & $9 \cdot 4-11 \cdot 8$ & $<0.001$ \\
\hline Skin folds subscapular (mm) & 230 & $6 \cdot 3$ & $5 \cdot 5-7 \cdot 2$ & $5 \cdot 8$ & $5 \cdot 2-6 \cdot 4$ & $6 \cdot 1$ & $5 \cdot 4-6 \cdot 7$ & $6 \cdot 3$ & $5 \cdot 7-7 \cdot 2$ & $7 \cdot 7$ & $6 \cdot 3-8 \cdot 7$ & $<0.001$ \\
\hline Infant feeding & & & & & & & & & & & & \\
\hline Days of exclusive BF & 233 & 126 & $91-152$ & 125 & $122-152$ & 135 & $117-166$ & 122 & $61-143$ & 129 & $61-152$ & 0.20 \\
\hline Fully BF at 4 months & 233 & & & & & & & & & & & 0.028 \\
\hline$n$ & & & 8 & & & & & & & & & \\
\hline$\%$ & & & 8 & & & & & & .9 & & & \\
\hline Days of any BF & 230 & 274 & $183-349$ & 304 & $212-357$ & 284 & $183-365$ & 227 & $152-318$ & 265 & $196-365$ & 0.98 \\
\hline Partial BF at 9 months & 233 & & & & & & & & & & & 0.31 \\
\hline$n$ & & & 9 & & & & & & & & & \\
\hline$\%$ & & & & & & & & & & & & \\
\hline Formula at 9 months $(\mathrm{g} / \mathrm{d})$ & 233 & 169 & $0-410$ & 93 & $0-334$ & 169 & $7-401$ & 269 & $11-449$ & 138 & $0-447$ & 0.15 \\
\hline Introduction to solids (months) & 233 & 4.0 & $4 \cdot 0-5 \cdot 0$ & 4.0 & $4 \cdot 0-5 \cdot 0$ & 4.0 & $4 \cdot 0-5 \cdot 0$ & $4 \cdot 0$ & $4 \cdot 0-5 \cdot 0$ & 4.0 & $4 \cdot 0-5 \cdot 0$ & 0.95 \\
\hline
\end{tabular}

FM, fat mass; FFM, fat-free mass; BF, breastfed.

*Trends across FMI quartiles were assessed using linear regression models adjusted for sex. † Not adjusted for sex. 
Table 4. Characteristics of the Småbørns Kost og Trivsel (SKOT) children according to fat-free mass index (FFMl, $\left.\mathrm{kg} / \mathrm{m}^{2}\right)$ quartile at 3 years of age* (Mean values and standard deviations, medians and interquartile ranges (IQR) (if not normally distributed), number of participants and percentages)

\begin{tabular}{|c|c|c|c|c|c|c|c|c|c|c|c|c|}
\hline & \multirow[b]{3}{*}{$n$} & & & \multicolumn{8}{|c|}{ 3-Year FFMI quartile } & \multirow[b]{3}{*}{$P$ for trend } \\
\hline & & \multicolumn{2}{|c|}{ Overall } & \multicolumn{2}{|c|}{$1(n 57)$} & \multicolumn{2}{|c|}{$2(n 59)$} & \multicolumn{2}{|c|}{$3(n 59)$} & \multicolumn{2}{|c|}{$4(n 58)$} & \\
\hline & & Median & IQR & Median & IQR & Median & IQR & Median & IQR & Median & IQR & \\
\hline \multicolumn{13}{|l|}{ Anthropometric characteristics } \\
\hline $\mathrm{FM}$ in girls $(\mathrm{kg}) \dagger$ & 122 & & & & & & & & & & & $<0.001$ \\
\hline Mean & & \multicolumn{2}{|c|}{$2 \cdot 71$} & \multicolumn{2}{|c|}{$2 \cdot 37$} & \multicolumn{2}{|c|}{2.46} & \multicolumn{2}{|c|}{$2 \cdot 78$} & \multicolumn{2}{|c|}{3.24} & \\
\hline SD & & \multicolumn{2}{|c|}{$0 \cdot 74$} & \multicolumn{2}{|c|}{0.59} & \multicolumn{2}{|c|}{0.62} & \multicolumn{2}{|c|}{0.65} & \multicolumn{2}{|c|}{0.76} & \\
\hline FM in boys $(\mathrm{kg}) \dagger$ & 111 & & & & & & & & & & & $<0.001$ \\
\hline Mean & & \multicolumn{2}{|c|}{$2 \cdot 29$} & \multicolumn{2}{|c|}{1.99} & \multicolumn{2}{|c|}{$2 \cdot 28$} & & & \multicolumn{2}{|c|}{$2 \cdot 69$} & \\
\hline SD & & \multicolumn{2}{|c|}{0.65} & \multicolumn{2}{|c|}{0.48} & \multicolumn{2}{|c|}{0.51} & & & \multicolumn{2}{|c|}{0.59} & \\
\hline FFM in girls $(\mathrm{kg}) \dagger$ & 122 & \multirow{2}{*}{\multicolumn{2}{|c|}{11.65}} & & & & & & & & & $<0.001$ \\
\hline Mean & & & & & & & 31 & & & & & \\
\hline SD & & & & & 35 & & 32 & & & & & \\
\hline FFM in boys $(\mathrm{kg}) \dagger$ & 111 & & & & & & & & & & & $<0.001$ \\
\hline Mean & & & & & 03 & & 49 & & & & & \\
\hline SD & & & & & & & 33 & & & & & \\
\hline BMI $\left(\mathrm{kg} / \mathrm{m}^{2}\right)$ & 233 & $15 \cdot 8$ & $15 \cdot 1-16 \cdot 6$ & $14 \cdot 8$ & $14 \cdot 4-15 \cdot 2$ & $15 \cdot 6$ & $15 \cdot 2-16 \cdot 0$ & $15 \cdot 9$ & $15 \cdot 5-16 \cdot 6$ & $17 \cdot 3$ & $16 \cdot 7-17.9$ & $<0.001$ \\
\hline Skin folds triceps $(\mathrm{mm})$ & 224 & $9 \cdot 3$ & $8 \cdot 1-10 \cdot 4$ & 8.6 & $7.7-9.9$ & $9 \cdot 0$ & $8 \cdot 0-10 \cdot 2$ & 9.4 & $8 \cdot 3-10 \cdot 7$ & $9 \cdot 7$ & $8 \cdot 8-11 \cdot 1$ & $<0.001$ \\
\hline Skin folds subscapular (mm) & 230 & $6 \cdot 3$ & $5 \cdot 5-7 \cdot 2$ & 5.9 & $5 \cdot 2-6 \cdot 7$ & $6 \cdot 3$ & $5 \cdot 4-6 \cdot 9$ & 6.4 & $5 \cdot 7-7 \cdot 4$ & 6.9 & $5 \cdot 9-8 \cdot 2$ & $<0.001$ \\
\hline Infant feeding & & & & & & & & & & & & \\
\hline Days of exclusive BF & 233 & 126 & $91-152$ & 132 & $105-152$ & 136 & $115-152$ & 122 & $44-152$ & 122 & $75-152$ & 0.24 \\
\hline Fully BF at 4 months & 233 & & & & & & & & & & & 0.26 \\
\hline$n$ & & & 8 & & & & 3 & & & & & \\
\hline$\%$ & & & & & .7 & & $\cdot 1$ & & & & & \\
\hline Days of any BF & 230 & 274 & $183-349$ & 274 & $183-335$ & 318 & $197-365$ & 244 & $173-335$ & 274 & $183-365$ & 0.99 \\
\hline Partial BF at 9 months & 233 & & & & & & & & & & & 0.19 \\
\hline$n$ & & & & & & & & & & & & \\
\hline$\%$ & & & & & 3 & & 3 & & & & & \\
\hline Formula at 9 months $(\mathrm{g} / \mathrm{d})$ & 233 & 169 & $0-410$ & 253 & $14-429$ & 89 & $0-316$ & 231 & $0-432$ & 146 & $0-422$ & 0.83 \\
\hline Introduction to solids (months) & 233 & 4.0 & $4 \cdot 0-5 \cdot 0$ & $4 \cdot 0$ & $4 \cdot 0-5 \cdot 0$ & 4.0 & $4 \cdot 0-5 \cdot 0$ & 4.0 & $4 \cdot 0-5 \cdot 0$ & 4.0 & $4 \cdot 0-5 \cdot 0$ & 0.52 \\
\hline
\end{tabular}

FM, fat mass; FFM, fat-free mass; $B F$, breastfed.

${ }^{*}$ Trends across FFMI quartiles were assessed using linear regression models adjusted for sex. † Not adjusted for sex. 
growth in the first 5 months of life are strong predictors of body composition at 3 years of age. A birth weight above $4000 \mathrm{~g}$ increased the probability of being in the highest quartile of FMI and FFMI at 3 years of age by approximately $50 \%$, compared to children with birth weights ranging from 3000 to $3500 \mathrm{~g}$. Rapid weight gain above $0.67 z$-scores from 0 to 5 months was strongly related to FMI, but not FFMI. Other studies have also found rapid early weight gain being associated with FM rather than $\mathrm{FFM}^{(3-5,40-42)}$, though studies on the effect of birth weight on later body composition support a stronger association with FFM than with $\mathrm{FM}^{(43,44)}$. It is possible that low birth weight is particularly responsible for a smaller proportion of FFM later in life ${ }^{(43)}$. However, one reason why we found birth weight to be equally related to FFM and FM could be due to the very small number of children with birth weights below $2500 \mathrm{~g}$ in this cohort.

Length measures of the children at 0 and 5 months resulted in very high HAZ scores, most likely due to a tendency on the part of midwives and general medical practitioners to overestimate length measurements in the primary healthcare sector, as has been demonstrated by other studies ${ }^{(45)}$. The WAZ values were found to be above the WHO standards at all ages. Disregarding WFH and BAZ at birth and 5 months, those values were also found to be above the WHO standards at 9, 18 and 36 months of age. Part of the explanation for this is that the cohort of the present study included infants who had only been breastfed for a short period. However, we have previously shown that the WAZ and BAZ of children from the SKOT cohort who were breastfed at 9 months were closer to the median of the WHO growth standards at 9 and 18 months or less compared to children who were no longer breastfed at 9 months $^{(32)}$. However, duration of full or partial breastfeeding was not found to be directly associated with BMI, FMI or FFMI at 3 years of age in the present study.

\section{Early feeding patterns as effect modifiers}

The effect of birth weight on FMI at 3 years of age was eliminated if the child had been fully breastfed for 6 months, as no effect modification was found for less than 6 months' duration of full breastfeeding. Duration of full breastfeeding was found to have a modifying effect on the impact of early growth on FMI at 3 years of age, although this effect modification was only of borderline significance for $1-3$ and 4-5 months. These findings indicate that, apart from the direct effect of breastfeeding on early growth ${ }^{(11,12,32)}$, a longer duration of full breastfeeding directly influences the extent to which early rapid weight gain adversely affects FM development. The Dortmund Nutritional and Anthropometric Longitudinally Designed Study (DONALD) ${ }^{(46)}$ showed a similar protective effect of full breastfeeding for at least 4 months with respect to body fat percentage among 249 infants, ranging from 2 to 5 years of age with early rapid weight gain. Preliminary data from the Promotion of Breastfeeding Intervention Trial (PROBIT) also showed a strong relationship between early rapid weight gain and obesity at 6-7 years of age, to be higher among children who had been exclusively formulafed from 1 month of age, compared to other infants ${ }^{(47)}$. 

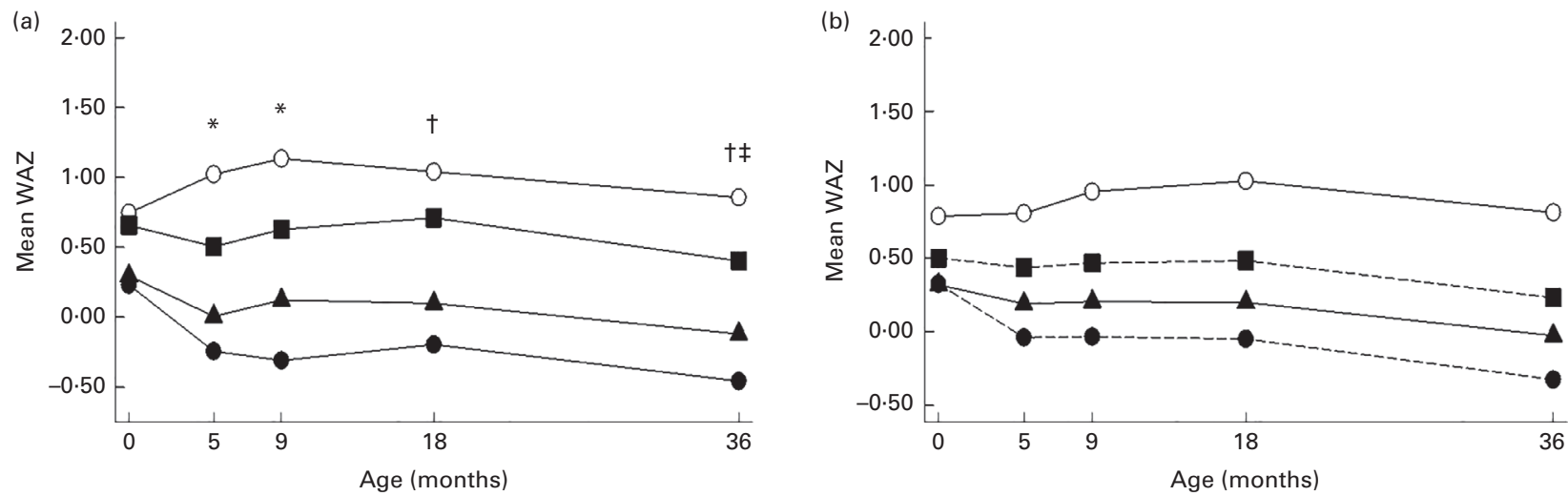

Fig. 1. Change in weight-for-age $z$-scores (WAZ) from 0 to 36 months according to (a) fat mass index (FMI) and (b) fat-free mass index (FFMI) quartiles at 3 years of age. - First quartile; 1 , second quartile; $n$, third quartile; $O$, fourth quartile. Differences between quartiles were assessed via mixed model analyses controlling for the educational level of the mother, smoking during pregnancy, gestational weight gain, household income and parental BMI. * Mean value of the fourth quartile (reference) was significantly higher than that of all other quartiles $(P<0.05)$. † Mean value of the fourth quartile was significantly higher than that of the first and second quartiles $(P<0.002)$. ¥Mean value of the fourth quartile was borderline higher than that in the third quartile $(P<0.08)$.

As the present study is observational, we cannot say for certain whether the effect modifications observed can be explained by physiology, decisions made by parents or advice given by healthcare workers. Also unrecognised confounders might still be operating. The duration of full breastfeeding and age of introduction to solids are by nature interdependent. Infants in the SKOT cohort partially breastfed still at 9 months had been introduced to complementary foods later than non-breastfed infants at 9 months ${ }^{(37)}$. Moreover, at this point of time, non-breastfed infants in this cohort had a higher intake of protein in their complementary diet, compared to partially breastfed infants ${ }^{(37)}$ who were also found to modify the effect of early growth on FM development ${ }^{(46)}$. The fact that we did not see a direct association between early feeding and FMI could be due to the insufficient power and the low number of overweight children in the present study.

The relationships between FFMI, birth weight and early growth were not affected by infant nutrition in the present study. Thus, we hypothesise that factors in pregnancy, the child's level of physical activity and dietary quality beyond infancy have a greater impact on FFMI.

\section{Strengths and limitations}

In the present study, FM and FFM were predicted using predictive equations generated from a large number of DXA scans in the same cohort. This was done to facilitate inclusion of more children in the analyses, as we had acceptable DXA scans from only 101 children and bioelectrical impedances of 233 children $^{(36)}$. Compared with FFM and FM measured by DXA, the predicted FFM and FM showed a prediction error of $2.8 \%(333 \mathrm{~g})$ for $\mathrm{FFM}$, and a prediction error of $11.3 \%(284 \mathrm{~g})$ for $\mathrm{FM}^{(36)}$. The observed associations are based on body composition of 3 -year-old children, and it is possible that the effect modifications of infant feeding on early growth observed in the present study do not persist beyond childhood. However, we surmise that FMI of 3-yearold children is a good predictor of later risk of obesity.

Table 6. Prevalence of children in the fourth quartile of fat mass index $\left(\mathrm{FMI}, \mathrm{kg} / \mathrm{m}^{2}\right)$ and fat-free mass index (FFMI, $\mathrm{kg} / \mathrm{m}^{2}$ ) according to birth weight and weight-for-age $z$-score change ( $\triangle \mathrm{WAZ}$ ) from 0 to 5 months (Number of participants and percentages)

\begin{tabular}{|c|c|c|c|c|c|c|c|}
\hline & \multirow[b]{2}{*}{ Total $n$} & \multicolumn{2}{|c|}{$\begin{array}{c}n \text { placed in } \\
\text { fourth quartile } \\
\text { of FMI }\end{array}$} & \multirow[b]{2}{*}{$P^{\star}$} & \multicolumn{2}{|c|}{$\begin{array}{l}n \text { placed in } \\
\text { fourth quartile } \\
\text { of FFMI }\end{array}$} & \multirow[b]{2}{*}{$P^{\star}$} \\
\hline & & $n$ & $\%$ & & $n$ & $\%$ & \\
\hline \multicolumn{8}{|l|}{ Birth weight (g) } \\
\hline$<3000$ & 23 & 5 & $21 \cdot 7$ & $0 \cdot 17 \dagger$ & 3 & $13 \cdot 0$ & $0.039+$ \\
\hline $3000-3499$ & 92 & 16 & $17 \cdot 4$ & 0.007 & 16 & $17 \cdot 4$ & 0.007 \\
\hline $3500-3999$ & 83 & 23 & $27 \cdot 7$ & 0.19 & 25 & $30 \cdot 1$ & 0.30 \\
\hline$\geq 4000$ & 35 & 14 & $40 \cdot 0$ & Reference & 14 & $40 \cdot 0$ & Reference \\
\hline \multicolumn{8}{|c|}{$\Delta$ WAZ (0-5 months) } \\
\hline$<-0.67$ & 49 & 7 & $14 \cdot 3$ & 0.001 & 10 & $20 \cdot 4$ & 0.47 \\
\hline$-0.67-0$ & 41 & 6 & $14 \cdot 6$ & 0.001 & 7 & $17 \cdot 1$ & 0.29 \\
\hline $0-0.67$ & 40 & 10 & $25 \cdot 0$ & 0.031 & 9 & $22 \cdot 5$ & 0.65 \\
\hline$>0.67$ & 37 & 18 & $48 \cdot 7$ & Reference & 10 & $27 \cdot 0$ & Reference \\
\hline
\end{tabular}

${ }^{*}$ Comparison of group difference against reference by Pearson's $\chi^{2}$ test. †Comparison by Fisher's exact test. 
Table 7. Multiple regression model of the association between birth weight, weight gain from 0 to 5 months, infant feeding and fat mass index (FMI) at 3 years of age $(n 156)^{*}$

( $\beta$ Coefficients and standard errors)

\begin{tabular}{lccc}
\hline Risk factor & $\beta$ & SE & $P$ \\
\hline Intercept & 2.68 & 0.48 & $<0.001$ \\
BWZ & 0.64 & 0.15 & $<0.001$ \\
$\Delta$ WAZ (0-5 months) & 0.73 & 0.17 & $<0.001$ \\
Sex (0, female; 1 , male) & -0.59 & 0.09 & $<0.001$ \\
Full breastfeeding & & & \\
$\quad<1$ month & Reference & & \\
$1-3$ months & -0.04 & 0.19 & 0.85 \\
$4-5$ months & -0.12 & 0.15 & 0.42 \\
6 months & 0.05 & 0.27 & 0.86 \\
Age of introduction & & & \\
3-4 months & Reference & & \\
5 months & 0.17 & 0.11 & 0.15 \\
6 months & 0.16 & 0.23 & 0.50 \\
BWZ $\times$ full breastfeeding & & & \\
$\quad<1$ month & Reference & & \\
$1-3$ months & -0.07 & 0.21 & 0.75 \\
$4-5$ months & -0.13 & 0.17 & 0.46 \\
6 months & -0.63 & 0.24 & 0.009 \\
$\Delta$ WAZ 0-5 months $\times$ full breastfeeding & & \\
$<1$ month & Reference & & \\
$1-3$ months & -0.30 & 0.20 & 0.13 \\
$4-5$ months & -0.30 & 0.18 & 0.10 \\
6 months & -0.77 & 0.29 & 0.010 \\
\hline
\end{tabular}

BWZ, birth weight $z$-score; WAZ, weight-for-age $z$-score.

${ }^{*} A$ multiple regression model with $\mathrm{FMl}\left(\mathrm{kg} / \mathrm{m}^{2}\right)$ at 3 years of age as the dependent variable controlling for BWZ, WAZ change from 0 to 5 months, educational level of mother, gestational weight gain and maternal BMI. The intercept represents the mean value of FMI at 3 years of age in the reference groups.

Other studies have shown a high level of tracking of FM from 2 to 7 years $^{(41)}$ and 4 to 9 years ${ }^{(48)}$, with a higher increase of FMI among those who acquired a high fat percentage earlier in life $\mathrm{e}^{(41,48)}$. A positive feature of the present study is the prospective and detailed information it obtained with regard to growth. The data of the study on the duration of breastfeeding and age of introduction to solids was collected retrospectively at 9 months. Unfortunately, the data on age of introduction to solids was crude (in months) and did not allow for a more detailed grouping of the ages of introduction. The main limitation of the present study is its small number of children fully breastfed for 6 months ( $n$ 21), as this group appears to drive the significant effect modifications. Breastfeeding is commonly associated with other beneficial life-style factors in western societies ${ }^{(19)}$, and with an observational study design, it will always be a risk to conjecture that the children breastfed for 6 months differ from children being breastfed for a shorter period of time with other characteristics.

In contrast to many other countries, full breastfeeding for 4-6 months is common in Denmark. Further, the families in the SKOT study are characterised by high education levels and high annual incomes, both the factors associated with longer durations of breastfeeding and later ages of introduction to solids ${ }^{(19)}$. In contrast to other studies ${ }^{(28,29)}$, most children in the SKOT study were introduced to solids at 4 months of age or later. Despite this relative homogeneity, infant feeding practices were found to modify the effect of early growth. The effect modifications of early feeding are likely to depend on alternatives to breastfeeding that differ from country to country. The infant feeding practices observed in the SKOT cohort are likely to differ from other populations worldwide. We desire that further studies be undertaken to investigate similar effect modifications of infant feeding on early growth that might lead to a global generalisation of the findings of the present study, which might be applicable to populations of different characteristics.

\section{Conclusion}

In conclusion, the present study found that birth weight and weight gain in the first 5 months of life had a strong influence on later body composition both in terms of FM and FFM. None of the other periods, from 5 months to 3 years of
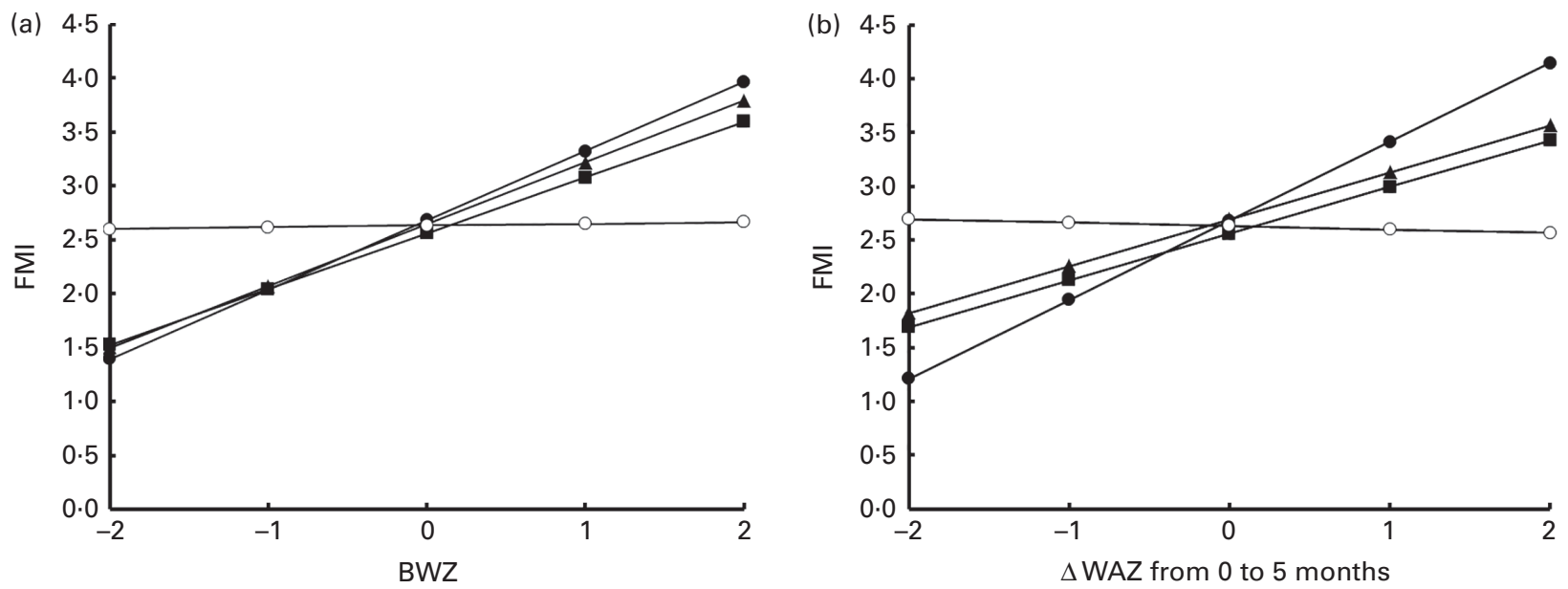

Fig. 2. Predicted mean fat mass index $\left(\mathrm{FMI}, \mathrm{kg} / \mathrm{m}^{2}\right.$ ) at 3 years of age according to (a) birth weight $z$-score (BWZ) and (b) change in weight-for-age $z$-scores ( $\triangle$ WAZ) from 0 to 5 months categorised into subgroups of breastfeeding duration in 156 children in the Småbørns Kost og Trivsel (SKOT) cohort. Figure lines are drawn from the coefficients in question derived from the multiple regression model presented in Table 7. (a) Illustrates the interaction between BWZ and duration of full breastfeeding and (b) illustrates the interaction between $\Delta$ WAZ from 0 to 5 months and duration of full breastfeeding. Duration of full breastfeeding $<1$ month (•, $n$ 36), 1-3 months $(\Lambda, n 39), 4-5$ months $(\square, n 137)$ and 6 months $(0, n 21)$. 
age, showed significant relations with FM and FFM at 3 years of age. Moreover, we have demonstrated that full breastfeeding for 6 months eliminated the impact on FMI at 3 years of age of both high birth weight and early growth in the first 5 months. Since the results are based on an observational study, causal effects can only be conjectures. However, the complex relation suggests that breastfeeding could have an additional protective effect apart from a direct effect on early growth. Thus, if infants with high birth weight or with rapid weight gain during the first few months are still fully breastfed, it seems reasonable to encourage the mother to continue full breastfeeding for about 6 months. Infant feeding was found not to modify the effect of early growth on FFMI. The results of our investigation suggest that early feeding can be important to modify the effect of high birth weight and excessive weight gain in early infancy, and support current recommendations concerning the duration of full breastfeeding.

\section{Acknowledgements}

We are very grateful to the children and families of the SKOT cohort for their involvement in the study. We are thankful as well to Anja Lykke Madsen and Laurine Bente Schram Harsløf, technical staff of the Department of Nutrition, Exercise and Sports, for their involvement in the data collection.

The SKOT study was supported by grants from The Danish Directorate for Food, Fisheries and Agri Business as part of the 'Complementary and young child feeding (CYCF) - impact on short- and long-term development and health' project (to K. F. M.). The Danish Directorate for Food, Fisheries and Agri Business had no role in the design, analysis, findings or writing of this article.

The authors' contributions are as follows: the study was designed by K. F. M., C. M., and K. T. E., and L. B. C. conducted the 3-year data collection examination. K. T. E., K. F. M., and C. M. formulated the research question. K. T. E. analysed the data and wrote the first draft of the manuscript. S. M. J. and C. R. supervised the quality standards of the statistical analyses. All authors contributed to the interpretation of the results and commented on the drafts. K. T. E. has primary responsibility for the final content. All authors have read and approved the final manuscript.

None of the authors has any conflicts of interest to declare.

\section{References}

1. Druet C, Stettler N, Sharp S, et al. (2012) Prediction of childhood obesity by infancy weight gain: an individual-level meta-analysis. Paediatr Perinat Epidemiol 26, 19-26.

2. Baird J, Fisher D, Lucas P, et al. (2005) Being big or growing fast: systematic review of size and growth in infancy and later obesity. BMJ 331, 929.

3. Singhal A, Kennedy K, Lanigan J, et al. (2010) Nutrition in infancy and long-term risk of obesity: evidence from 2 randomized controlled trials. Am J Clin Nutr 92, 1133-1144.

4. Chomtho S, Wells JC, Williams JE, et al. (2008) Infant growth and later body composition: evidence from the 4-component model. Am J Clin Nutr 87, 1776-1784.
5. Ekelund U, Ong K, Linne Y, et al. (2006) Upward weight percentile crossing in infancy and early childhood independently predicts fat mass in young adults: the Stockholm Weight Development Study (SWEDES). Am J Clin Nutr 83, 324-330.

6. Stettler N, Zemel BS, Kumanyika S, et al. (2002) Infant weight gain and childhood overweight status in a multicenter, cohort study. Pediatrics 109, 194-199.

7. Dennison BA, Edmunds LS, Stratton HH, et al. (2006) Rapid infant weight gain predicts childhood overweight. Obesity (Silver Spring) 14, 491-499.

8. Taveras EM, Rifas-Shiman SL, Belfort MB, et al. (2009) Weight status in the first 6 months of life and obesity at 3 years of age. Pediatrics 123, 1177-1183.

9. Lagstrom H, Hakanen M, Niinikoski H, et al. (2008) Growth patterns and obesity development in overweight or normalweight 13-year-old adolescents: the STRIP study. Pediatrics 122, e876-e883.

10. Victora CG, Sibbritt D, Horta BL, et al. (2007) Weight gain in childhood and body composition at 18 years of age in Brazilian males. Acta Paediatr 96, 296-300.

11. Dewey KG (1998) Growth characteristics of breast-fed compared to formula-fed infants. Biol Neonate 74, 94-105.

12. Victora CG, Morris SS, Barros FC, et al. (1998) The NCHS reference and the growth of breast- and bottle-fed infants. J Nutr 128, 1134-1138.

13. Gale C, Logan KM, Santhakumaran S, et al. (2012) Effect of breastfeeding compared with formula feeding on infant body composition: a systematic review and meta-analysis. Am J Clin Nutr 95, 656-669.

14. Arenz S, Ruckerl R, Koletzko B, et al. (2004) Breast-feeding and childhood obesity - a systematic review. Int $J$ Obes Relat Metab Disord 28, 1247-1256.

15. Harder T, Bergmann R, Kallischnigg G, et al. (2005) Duration of breastfeeding and risk of overweight: a meta-analysis. Am J Epidemiol 162, 397-403

16. Owen CG, Martin RM, Whincup PH, et al. (2005) Effect of infant feeding on the risk of obesity across the life course: a quantitative review of published evidence. Pediatrics 115, 1367-1377.

17. Owen CG, Martin RM, Whincup PH, et al. (2005) The effect of breastfeeding on mean body mass index throughout life: a quantitative review of published and unpublished observational evidence. Am J Clin Nutr 82, 1298-1307.

18. Robinson SM, Marriott LD, Crozier SR, et al. (2009) Variations in infant feeding practice are associated with body composition in childhood: a prospective cohort study. J Clin Endocrinol Metab 94, 2799-2805.

19. Toschke AM, Martin RM, von Kries R, et al. (2007) Infant feeding method and obesity: body mass index and dual-energy X-ray absorptiometry measurements at 9-10 y of age from the Avon Longitudinal Study of Parents and Children (ALSPAC). Am J Clin Nutr 85, 1578-1585.

20. Yin J, Quinn S, Dwyer T, et al. (2012) Maternal diet, breastfeeding and adolescent body composition: a 16-year prospective study. Eur J Clin Nutr 66, 1329-1334.

21. Gale CR, Javaid MK, Robinson SM, et al. (2007) Maternal size in pregnancy and body composition in children. J Clin Endocrinol Metab 92, 3904-3911.

22. Butte NF, Wong WW, Hopkinson JM, et al. (2000) Infant feeding mode affects early growth and body composition. Pediatrics 106, 1355-1366.

23. Burdette HL, Whitaker RC, Hall WC, et al. (2006) Breastfeeding, introduction of complementary foods, and adiposity at 5 y of age. Am J Clin Nutr 83, 550-558. 
24. Tulldahl J, Pettersson K, Andersson SW, et al. (1999) Mode of infant feeding and achieved growth in adolescence: early feeding patterns in relation to growth and body composition in adolescence. Obes Res 7, 431-437.

25. Moorcroft KE, Marshall JL \& McCormick FM (2011) Association between timing of introducing solid foods and obesity in infancy and childhood: a systematic review. Matern Child Nutr 7, 3-26.

26. Przyrembel H (2012) Timing of introduction of complementary food: short- and long-term health consequences. Ann Nutr Metab 60, Suppl. 2, 8-20.

27. Grote V, Schiess SA, Closa-Monasterolo R, et al. (2011) The introduction of solid food and growth in the first $2 \mathrm{y}$ of life in formula-fed children: analysis of data from a European cohort study. Am J Clin Nutr 94, 1785S-1793S.

28. Ong KK, Emmett PM, Noble S, et al. (2006) Dietary energy intake at the age of 4 months predicts postnatal weight gain and childhood body mass index. Pediatrics 117, e503-e 508

29. Tarrant RC, Younger KM, Sheridan-Pereira M, et al. (2010) Factors associated with weaning practices in term infants: a prospective observational study in Ireland. BrJ Nutr 104, $1544-1554$

30. Wells JC (2000) A Hattori chart analysis of body mass index in infants and children. Int J Obes Relat Metab Disord 24 , 325-329.

31. Madsen AL, Schack-Nielsen L, Larnkjaer A, et al. (2010) Determinants of blood glucose and insulin in healthy 9-month-old term Danish infants; the SKOT cohort. Diabet Med 27, 1350-1357.

32. Madsen AL, Larnkjaer A, Molgaard C, et al. (2011) IGF-I and IGFBP-3 in healthy 9 month old infants from the SKOT cohort: breastfeeding, diet, and later obesity. Growth Horm IGF Res 21, 199-204.

33. World Health Organization (2011) WHO Anthro (version 3.2.2, January 2011) and macros. http://www.who.int/ childgrowth/software/en/ (accessed 4 March 2013).

34. Cole TJ, Bellizzi MC, Flegal KM, et al. (2000) Establishing a standard definition for child overweight and obesity worldwide: international survey. BMJ 320, 1240-1243.

35. World Health Organization (2006) WHO child growth standards: length/height-for-age, weight-for-age, weightfor-length, weight-for-height and body mass index-for-age: methods and development. http://www.who.int/child growth/standards/en/ (accessed 14 February 2013).

36. Ejlerskov KT, Jensen SM, Christensen LB, et al. (2014) Prediction of fat-free body mass from bioelectrical impedance and anthropometry among 3-year-old children using DXA Sci Rep 4, 3889.

37. Gondolf UH, Tetens I, Michaelsen KF, et al. (2012) Dietary habits of partly breast-fed and completely weaned infants at 9 months of age. Public Health Nutr 15, 578-586.

38. Wells JC (2001) A critique of the expression of paediatric body composition data. Arch Dis Child 85, 67-72.

39. Ong KK \& Loos RJ (2006) Rapid infancy weight gain and subsequent obesity: systematic reviews and hopeful suggestions. Acta Paediatr 95, 904-908.

40. Larnkjaer A, Schack-Nielsen L, Molgaard C, et al. (2010) Effect of growth in infancy on body composition, insulin resistance, and concentration of appetite hormones in adolescence. Am J Clin Nutr 91, 1675-1683.

41. Karaolis-Danckert N, Buyken AE, Bolzenius K, et al. (2006) Rapid growth among term children whose birth weight was appropriate for gestational age has a longer lasting effect on body fat percentage than on body mass index. Am J Clin Nutr 84, 1449-1455.

42. Ong KK, Emmett $\mathrm{P}$, Northstone K, et al. (2009) Infancy weight gain predicts childhood body fat and age at menarche in girls. J Clin Endocrinol Metab 94, $1527-1532$.

43. Singhal A, Wells J, Cole TJ, et al. (2003) Programming of lean body mass: a link between birth weight, obesity, and cardiovascular disease? Am J Clin Nutr 77, 726-730.

44. Wells JC, Chomtho S \& Fewtrell MS (2007) Programming of body composition by early growth and nutrition. Proc Nutr Soc 66, 423-434.

45. Rifas-Shiman SL, Rich-Edwards JW, Scanlon KS, et al. (2005) Misdiagnosis of overweight and underweight children younger than 2 years of age due to length measurement bias. MedGenMed 7, 56 .

46. Karaolis-Danckert N, Gunther AL, Kroke A, et al. (2007) How early dietary factors modify the effect of rapid weight gain in infancy on subsequent body-composition development in term children whose birth weight was appropriate for gestational age. Am J Clin Nutr 86, 1700-1708.

47. Manasseh Z, Kramer MS \& Dewey KG (2010) Infant feeding modifies the relationship between rapid weight gain in infancy and childhood obesity (abstract). FASEB J. http:// www.fasebj.org/cgi/content/meeting_abstract/24/1_Meeting Abstracts/556.5.

48. Goulding A, Taylor RW, Jones IE, et al. (2003) Body composition of 4- and 5-year-old New Zealand girls: a DXA study of initial adiposity and subsequent 4-year fat change. Int J Obes Relat Metab Disord 27, 410-415. 